\title{
Expansions and Phase Transitions for the Ground State of Quantum Ising Lattice Systems
}

\author{
James R. Kirkwood ${ }^{1,2}$ and Lawrence E. Thomas ${ }^{3}$ \\ Department of Mathematics, University of Virginia, Charlottesville, VA 22903, USA
}

\begin{abstract}
Expansions for the ground state of some transverse Ising-like models are developed. These expansions are easily estimated by the solutions to some simple implicit equations. Short range or long range order obtains, depending on the coupling constants of the models.
\end{abstract}

\section{Introduction}

Let $H_{\Lambda}(\boldsymbol{\varepsilon})$ be a quantum lattice Hamiltonian associated with a finite volume $\Lambda \subset \mathbb{Z}^{v}$ and analytically dependent on coupling constants $\boldsymbol{\varepsilon}=\left(\varepsilon_{1}, \ldots, \varepsilon_{n}\right)$. Let $\psi_{\Lambda}(\boldsymbol{\varepsilon})$ be the corresponding normalized ground state eigenvector, and formally let

$$
\varrho(\cdot ; \varepsilon)=\lim _{\Lambda \uparrow \mathbb{Z}^{v}}\left\langle\psi_{\Lambda}(\boldsymbol{\varepsilon}),(\cdot) \psi_{\Lambda}(\boldsymbol{\varepsilon})\right\rangle
$$

be the corresponding state defined by the $\psi_{\Lambda}(\varepsilon)$ 's in the thermodynamic limit. The purpose of this article is to show that $\varrho(\varepsilon)$, as a function of $\varepsilon$, can exhibit short or long range order, i.e. that $\varrho(\varepsilon)$ undergoes a phase transition in $\boldsymbol{\varepsilon}$ in all dimensions $v \geqq 1$, at least for a particularly simple family of Hamiltonians $H_{A}(\boldsymbol{\varepsilon})$.

The Hamiltonians we consider, transverse Ising models, which we assume to depend on two parameters $\varepsilon=(\varepsilon, \delta)$, are defined by

$$
-H_{\Lambda}(\varepsilon, \delta)=\sum_{i \in \Lambda}\left(1+\delta K\left(i, \sigma^{z}\right)\right) \sigma^{x}(i)+\varepsilon \sum_{A \subset A} \hat{V}(A) \sigma^{z}(A) .
$$

Here, $\sigma^{x}(i)$ and $\sigma^{z}(i)$ are the usual Pauli spin matrices acting at the site $i$,

$$
\sigma^{x}(i)=\left(\begin{array}{ll}
0 & 1 \\
1 & 0
\end{array}\right), \quad \sigma^{z}(i)=\left(\begin{array}{rr}
1 & 0 \\
0 & -1
\end{array}\right)
$$

1 Now at Institute for Mathematics and its Applications, Minneapolis, MN 55455, USA

2 Research in partial fulfillment of the requirements for the $\mathrm{Ph}$. $\mathrm{D}$. degree at the University of Virginia

3 Work partially supported by NSF-MCS 74-07313-A03 
taken in a basis so that the first term of (1.2) is a spin-flip term, the second term a multiplication (Ising) term. The $\hat{V}(A)$ are real coefficients, $\sigma^{z}(A)=\bigotimes_{i \in A} \sigma^{z}(i)$, and the $K\left(i, \sigma^{z}\right)$ are functions of the $\sigma^{z}$ s [and such that $H_{\Lambda}(\varepsilon, \delta)$ is selfadjoint]. For simplicity of the analysis we assume throughout that $H_{\Lambda}(\varepsilon, \delta)$ is translation invariant; in particular, we assume that $\Lambda$ is rectangular in shape and that in Eq. (1.2) periodic boundary conditions are imposed.

There are two reasons for investigating the correlations of $\psi_{\Lambda}(\varepsilon, \delta)$ and $\varrho_{\Lambda}(\varepsilon, \delta)$. First, one expects $\varrho_{\Lambda}(\varepsilon, \delta)$ to approximate the extremely low temperature thermodynamic states corresponding to $H_{\Lambda}(\varepsilon, \delta)$ (spin-wave approximation). If, for example, $\varrho_{A}(\varepsilon, \delta)$ has only short range order, one might conjecture that the thermodynamic states do not undergo a phase transition at any temperature; in other words, quantum fluctuations coming from the spin-flip terms of $H_{\Lambda}(\varepsilon, \delta)$ are sufficiently strong in comparison with the Ising terms that long range order is not possible at any temperature.

The second reason for considering the correlations of $\psi_{A}(\varepsilon, \delta)$ is their relevance to dynamical phase transitions for Markov semigroups. We discuss this reason from a formal viewpoint. Let $X_{1}, \ldots, X_{N}$ be multiplication operators and define the (thermodynamic) state $\varrho_{\Lambda}(\varepsilon, \delta, \beta)$ by

$$
\begin{aligned}
& \varrho_{\Lambda}\left(X_{1}\left(t_{1}\right) \ldots X_{N}\left(t_{N}\right) ; \varepsilon, \delta, \beta\right) \\
& \quad=\left(\operatorname{tr} e^{-\beta H}\right)^{-1} \operatorname{tr}\left(e^{-t_{1} H_{\Lambda}} X_{1} e^{-\left(t_{2}-t_{1}\right) H_{\Lambda}} X_{2} \ldots e^{-\left(t_{N}-t_{N}-1\right) H_{\Lambda}} X_{N} e^{-\left(\beta-t_{N}\right) H_{\Lambda}}\right) .
\end{aligned}
$$

The state $\varrho_{\Lambda}(\varepsilon, \delta, \beta)$ defines [at least for $\delta$ sufficiently small so that $\left(1+\delta K\left(i, \sigma^{z}\right)\right)>0$ ] a periodic stochastic process [1] which becomes a Markov process in the $\beta \rightarrow \infty$ limit. The path space measure associated with the infinite $\beta$ state $\varrho_{\Lambda}(\varepsilon, \delta)$ clearly satisfies

$$
\varrho_{\Lambda}(X, \varepsilon, \delta)=\left\langle\psi_{\Lambda}(\varepsilon, \delta), X \psi_{\Lambda}(\varepsilon, \delta)\right\rangle,
$$

so that in the $\Lambda \uparrow \mathbb{Z}^{v}$ limit, the resulting infinite volume path space measure has short or long range order depending on $\varepsilon, \delta$, at least in spatial directions.

Technically, the proofs of short and long range order are simpler for $\varrho(\varepsilon, \delta)$ than for the thermodynamic states; but the proofs do present a novel feature. The idea is first to choose an orthonormal basis so that $\psi_{A}(\varepsilon, \delta)$ is represented as an $l^{2}$-function $\exp -\frac{1}{2} \phi_{\Lambda}(\sigma, \varepsilon, \delta), \sigma \in\{-1,1\}^{\Lambda}$. (By standard Perron-Frobenius arguments this function may be taken to be positive.) The function $\phi_{\Lambda}$ is then expanded in a power series in $\varepsilon, \delta$ with power series coefficients obtained successively by a nonlinear recursion relation. These coefficients are however easily estimated by the power series coefficients of functions defined implicitly by some (nonlinear) equations. Curiously, these implicit equations are essentially the same as those considered by Hagedorn and Rafelski [2] recently for rather different reasons.

Section II describes the small $\varepsilon, \delta$ expansion in detail from which short-range order follows. Section III describes a large $\varepsilon$-expansion and a proof of long range order, for the simple transverse nearest neighbor Ising model; this section is also intended to illustrate that more complicated flipping mechanisms besides the single site flips of Eq. (1.2) can be accommodated by the same techniques.

That the simple one-dimensional transverse Ising model has a phase transition in its ground state is well-known; cf. the article of Pfeuty [3] who proves the 
transition by exploiting the Jordan-Wigner transformation and the close relation between this model and the two-dimension anisotropic Ising model. Our results extend the proof of a transition to more general interactions and, of course, to higher dimensions. The expansions we obtain should be compared with those of Pfeuty and Elliot [4] and, particularly, those of Kadanoff and Kohmoto [5] who expand the ground states themselves rather than their logarithms. The logarithm expansions, we believe, lend themselves to investigating questions of short and long range behavior; moreover they are remarkably simple to estimate.

We also mention that an approach to phase transitions for the ground state, in principle, is to examine the $\beta \rightarrow \infty$ limit of the thermodynamic states as in Eq. (1.3) above (before taking the $\Lambda \uparrow \mathbb{Z}^{v}$ limit). For $v \geqq 2$ dimensions, the thermodynamic states can be analyzed via space-time Peierls contours, following Ginibre [6], to establish long range order. But the estimates fail for $\beta \rightarrow \infty$; effectively $\varepsilon$ must go to $\infty$ as well for these estimates to show long range order, an unphysical result. Alternatively, an argument of Lieb and Fröhlich [7] exploiting reflection positivity (when it applies) can be used to infer long range order for the thermodynamic states $(v \geqq 2)$ with $\varepsilon$ sufficiently large and fixed which is uniform in $\beta, \beta \rightarrow \infty$ and which therefore implies long range order for the ground state. A discussion of these points is contained in [8] and will be reported elsewhere.

Finally, we remark that the techniques described here can be adapted to obtain low temperature expansions for thermodynamic states which do not directly involve Trotter product approximations. This work, too, will be reported elsewhere.

\section{Small $\varepsilon, \delta$ Expansion}

We first introduce some notation. Let $\sigma$ denote a point (configuration) in $\{-1,1\}^{\Lambda}$; let $\sigma(i)$ be the coordinate of $\sigma$ at side $i \in \Lambda[\sigma(i)$ is just the usual Ising variable] and set $\sigma(A)=\prod_{i \in A} \sigma(i)$ for $A \subset \Lambda$. We denote by $\sigma_{i}$ the configuration obtained from $\sigma$ by flipping the spin at site $i$, i.e. $\sigma_{i}(k)=\sigma(k) k \neq i, \sigma_{i}(k)=-\sigma(k)$ $k=1$, and $\sigma_{i j}$ the configuration obtained from $\sigma$ by flipping at sites $i$ and $j$. As orthonormal basis for the Hilbert space $\bigotimes_{A} \mathbb{C}^{2}$ with the usual inner product, we take vectors of the form $|\sigma\rangle=\bigotimes_{i \in \Lambda}|\sigma(i)\rangle$ with $|\sigma(i)\rangle$ the state at site $i$ which is spin up or down with respect to $\sigma^{z}(i)$ according to whether $\sigma(i)=+1$ or -1 respectively.

As ansatz for the (unnormalized) ground state eigenfunction $\psi_{\Lambda}(\varepsilon, \delta)$ we take

$$
\psi_{\Lambda}(\varepsilon, \delta)=\sum_{\sigma} \psi_{\Lambda}(\sigma, \varepsilon, \delta)|\sigma\rangle
$$

with

$$
\psi_{\Lambda}(\sigma, \varepsilon, \delta)=\exp \left(-\frac{1}{2} \sum_{A \subset \Lambda} J_{\Lambda}(A, \varepsilon, \delta) \sigma(A)\right),
$$

with the constants $J_{A}$ to be determined from $\phi_{A}(i, \sigma, \varepsilon, \delta)$ defined below and

$$
\phi_{A}(i, \sigma, \varepsilon, \delta)=\sum_{\substack{A: \\ i \in A}} J_{\Lambda}(A, \varepsilon, \delta) \sigma(A) .
$$


Let $\|\cdot\|$ denote the $l^{1}$-norm of the Fourier transform of functions of $\sigma$, i.e. if

$$
\chi(\sigma)=\sum_{A} \hat{\chi}(A) \sigma(A)
$$

then

$$
\|\chi\|=\sum_{A}|\hat{\chi}(A)|
$$

Given a set $A \subset \Lambda$ we let range $A=\sup _{i, j \in A}|i-j|$, where $|i-j|$ is the minimal path length between $i, j$ computed along the bonds of $\mathbb{Z}^{v}$. Given a function $\chi(\sigma)$ with expansion (2.4), we set range $\chi=$ sup range $A$ with the sup over all $A$ such that $\hat{\chi}(A) \neq 0$. Finally, we let range $\chi$ based at $i$ be sup range $(A \cup\{i\})$ with sup again over all $A$ with $\hat{\chi}(A) \neq 0$.

Theorem 2.1. Assume $H_{A}(\varepsilon, \delta)$ defined by Eq. (1.2) is translationally invariant and assume that range $K(i)$ based at $i$ and range $\left(\sum_{i \in A} \hat{V}(A) \sigma(A)\right)$ are both $\leqq r$ for some $r<\infty$, independent of $\Lambda$. Then there are constants $c_{1}, c_{2}(\varepsilon, \delta), c_{3}(\varepsilon, \delta), \varepsilon_{0}, \delta_{0}$ such that for $\varepsilon<\varepsilon_{0}, \delta<\delta_{0}$,

(i) $\phi_{\Lambda}(i, \sigma, \varepsilon, \delta)$ is an absolutely convergent power series in $\varepsilon$ and $\delta$ and

$$
\left\|\phi_{\Lambda}(i, \cdot, \varepsilon, \delta)\right\|<c_{1} \sup (|\varepsilon|,|\delta|) .
$$

(ii) Further,

$$
\left\|\phi_{\Lambda}\left(i, \sigma_{j}, \varepsilon, \delta\right)-\phi_{\Lambda}(i, \sigma, \varepsilon, \delta)\right\| \leqq c_{2}(\varepsilon, \delta)(\sup (|\varepsilon|,|\delta|))^{|i-j| / r} .
$$

(iii) The estimates are uniform in $\Lambda$. In particular if $\Lambda \subset \Lambda^{\prime}$ and $i \in \Lambda$,

$$
\left\|\phi_{\Lambda}(i, \cdot, \varepsilon, \delta)-\phi_{\Lambda^{\prime}}(i, \cdot, \varepsilon, \delta)\right\| \leqq c_{3}(\varepsilon, \delta)\left(\sup (|\varepsilon|,|\delta|)^{d / r},\right.
$$

where $d$ is the distance between $i$ and the boundary of $\Lambda$.

Remark. Let $\mu_{\Lambda}(\varepsilon, \delta)$ be the Ising Gibbs measure defined by

$$
\mu_{\Lambda}(\{\sigma\}, \varepsilon, \delta)=\frac{1}{\mathbb{Z}_{\Lambda}(\varepsilon, \delta)} \psi_{\Lambda}^{2}(\sigma, \varepsilon, \delta),
$$

with

$$
\mathbb{Z}_{\Lambda}(\varepsilon, \delta)=\sum_{\sigma} \psi_{\Lambda}^{2}(\sigma, \varepsilon, \delta)=\left\langle\psi_{\Lambda}(\varepsilon, \delta), \psi_{\Lambda}(\varepsilon, \delta)\right\rangle
$$

and \langle\rangle the Hilbert space inner product. Using standard expansion methods [9, Chap. 4] and the above theorem, it follows that $\mu_{\Lambda}(\varepsilon, \delta)$ has a unique thermodynamic limit $\Lambda \uparrow \mathbb{Z}^{2}$ with the resulting measure having short range correlations, for $\varepsilon, \delta$ sufficiently small. In particular,

$$
\lim _{\Lambda \uparrow \mathbb{Z}^{v}} \frac{\left\langle\psi_{\Lambda}(\varepsilon, \delta), \sigma^{z}(i) \sigma^{z}(j) \psi_{\Lambda}(\varepsilon, \delta)\right\rangle}{\left\langle\psi_{\Lambda}(\varepsilon, \delta), \psi_{\Lambda}(\varepsilon, \delta)\right\rangle}=\lim _{\Lambda \uparrow \mathbb{Z}^{v}}\left(\mu_{\Lambda}(\varepsilon, \delta), \sigma(i) \sigma(j)\right)
$$

decays exponentially fast in $|i-j|$, and short range order obtains. 
Proof of the Theorem. The eigenvector equation

$$
-H_{\Lambda}(\varepsilon, \delta) \psi_{\Lambda}(\varepsilon, \delta)=|\Lambda| e_{\Lambda}(\varepsilon, \delta) \psi_{\Lambda}(\varepsilon, \delta)
$$

with $|\Lambda| e_{\Lambda}(\varepsilon, \delta)$ the eigenvalue, can be written in terms of $\psi_{\Lambda}(\sigma, \varepsilon, \delta)$ and then, in turn, after division by $\psi_{\Lambda}(\sigma, \varepsilon, \delta)$, in terms of the $\phi_{\Lambda}(i, \sigma, \varepsilon, \delta)$

$$
\sum_{i}\left(1+\delta K_{i}(\sigma)\right) \exp \phi_{\Lambda}(i, \sigma, \varepsilon, \delta)+\varepsilon \sum_{A \subset \Lambda} \hat{V}(A) \sigma(A)=|\Lambda| e_{\Lambda}(\varepsilon, \delta) .
$$

Expanding (and suppressing the $\Lambda$ subscript)

$$
\begin{aligned}
\phi_{\Lambda}(i, \sigma, \varepsilon, \sigma) & =\sum_{n=0}^{\infty} \varepsilon^{n} \phi_{n}(i, \sigma, \delta), \\
e_{\Lambda}(\varepsilon, \delta) & =\sum_{n=0}^{\infty} \varepsilon^{n} e_{n}(\delta),
\end{aligned}
$$

and equating powers of $\varepsilon$, we obtain

$$
\begin{gathered}
\sum_{i \in \Lambda}(1+\delta K(i, \sigma)) \exp \phi_{0}(i, \sigma, \delta)=|\Lambda| e_{0}(\delta), \\
\sum_{i \in \Lambda}(1+\delta K(i, \sigma))\left(\exp \phi_{0}(i, \sigma, \delta)\right) \phi_{1}(i, \sigma, \delta)+\sum_{A \subset \Lambda} \hat{V}(A) \sigma(A)=|\Lambda| e_{1}(\delta),
\end{gathered}
$$

and, otherwise,

$$
\begin{aligned}
\sum_{i \in \Lambda} & {\left[(1+\delta K(i, \sigma))\left(\exp \phi_{0}(i, \sigma, \delta)\right)\left(\phi_{n}(i, \sigma, \delta)+P_{n}\left(\phi_{1}(i, \sigma, \delta)\right), \ldots, \phi_{n-1}(i, \sigma, \delta)\right)\right] } \\
& =|\Lambda| e_{n}(\delta), \quad n \geqq 2,
\end{aligned}
$$

where $P_{n}\left(x_{1}, \ldots, x_{n-1}\right)$ is the polynomial defined by the generating functional

$$
\exp \left(\sum_{n=1}^{\infty} \varepsilon^{n} x_{n}\right)=1+\sum_{n=1}^{\infty} \varepsilon^{n}\left(x_{n}+P_{n}\left(x_{1}, \ldots, x_{n-1}\right)\right) .
$$

Note that $P_{n}$ has positive coefficients; by definition $P_{1}=0$.

To solve Eqs. $(2.8 \mathrm{a})-(2.8 \mathrm{c})$, we first note the important closure property, which follows from Eq. (2.3): If $\sigma(A)$ occurs in the Fourier expansion for $\phi_{\Lambda}(i, \sigma, \varepsilon, \delta)$ with coefficient $J_{\Lambda}(A, \varepsilon, \delta)$ and $j \in A$, then $\sigma(A)$ also occurs in the expansion for $\phi_{\Lambda}(j, \varepsilon, \delta)$ with the same coefficient $J_{\Lambda}(A, \varepsilon, \delta)$. In particular, if $\phi_{\Lambda}(i, \sigma, \varepsilon, \delta)$ and $\phi_{\Lambda}(j, \sigma, \varepsilon, \delta)$ are expanded in power series in $\varepsilon, \delta$, and $A$ contains $i$ and $j$, then the coefficient of $\delta^{m} \varepsilon^{n} \sigma(A)$ in both expansions will be equal.

We proceed to the solution of Eq. (2.8a). Writing

$$
\begin{aligned}
\phi_{0}(i, \sigma, \delta) & =\sum_{m=1}^{\infty} \delta^{m} \phi_{m, 0}(i, \sigma), \\
e_{0}(\delta) & =1+\sum_{m=1}^{\infty} \delta^{m} e_{m, 0},
\end{aligned}
$$

and substituting into Eq. (2.8a), we obtain

$$
\sum_{i \in \Lambda}\left(\phi_{1,0}(i, \sigma)+K(i, \sigma)\right)=|\Lambda| e_{1,0},
$$


and

$$
\begin{aligned}
\sum_{i \in \Lambda}\left(\phi_{m, 0}(i, \sigma)+P_{m}\left(\phi_{1.0}(i, \sigma), \phi_{2,0}(i, \sigma), \ldots, \phi_{m-1,0}(i, \sigma)\right)\right) \\
\quad+\sum_{i \in \Lambda} K(i, \sigma)\left(\phi_{m-1,0}(i, \sigma)+P_{m-1}\left(\phi_{1,0}(i, \sigma), \ldots, \phi_{m-2,0}(i, \sigma)\right)\right) \\
=|\Lambda| e_{m, 0}, \quad m \geqq 2 .
\end{aligned}
$$

Using the facts that $\phi(i, \sigma, \delta, \varepsilon)$ and hence $\phi_{m, 0}(i, \sigma, \delta, \varepsilon)$ are homogeneous in $\sigma(i)$, and the closure property, the reader can convince himself that these equations have a unique solution.

We next estimate the series Eqs. (2.9) and (2.10). It is convenient to define

$$
\chi_{m, 0}(i, \sigma)=\phi_{m .0}(i, \sigma)-e_{m, 0} .
$$

Note that by the closure property, translation invariance and the fact that the Fourier expansion for $\phi_{m, 0}(i, \sigma)$ contains no constant term, we have that

$$
\left\|\sum_{i} \chi_{m, 0}(i)\right\|=|\Lambda|\left\|\chi_{m, 0}(i)\right\|
$$

and

$$
\left\|\chi_{m, 0}(i)\right\|=\left\|\phi_{m, 0}(i)\right\|+\left|e_{m, 0}\right| .
$$

Using these estimates, Eqs. (2.11a) and (2.11b) give the estimates

$$
\begin{aligned}
\left\|\chi_{1,0}(i)\right\| \leqq & \|K(i)\|, \\
\left\|\chi_{m, 0}(i)\right\| \leqq & P_{m}\left(\left\|\chi_{1,0}(i)\right\|, \ldots,\left\|\chi_{m-1,0}(i)\right\|\right) \\
& \quad+\|K(i)\|\left(\left\|\chi_{m-1,0}(i)\right\|+P_{m-1}\left(\left\|\chi_{1,0}(i)\right\|, \ldots,\left\|\chi_{m-2,0}(i)\right\|\right)\right), \quad m \geqq 2 .
\end{aligned}
$$

Let $g(\delta)$ be the analytic function defined in a neighborhood of $\delta=0(g(0)=0)$ by the implicit relation

$$
G(g, \delta)=2 g-e^{g}+1-\|K(i)\| \delta e^{g}=0 .
$$

[The function $g$ exists since $\partial G / \partial g(0,0) \neq 0$.] Substituting

$$
g=\sum_{m=1}^{\infty} \delta^{m} g_{m}
$$

into the rearranged equation

$$
g=e^{g}-g-1+\|\left(K(i) \| \delta e^{g},\right.
$$

we get the nonlinear recursion relations

$$
\begin{aligned}
& g_{1}=\|K(i)\|, \\
& g_{m}=P_{m}\left(g_{1}, \ldots, g_{m-1}\right)+\left\|K_{i}\right\|\left(g_{m-1}+P_{m-1}\left(g_{1}, \ldots, g_{m-2}\right)\right), \quad m \geqq 2 .
\end{aligned}
$$

By induction,

$$
\left\|\chi_{m, 0}(i)\right\| \leqq g_{m}
$$


so that both series Eqs. (2.9) and (2.10) are absolutely convergent with bounds

$$
\begin{aligned}
\left\|\phi_{0}(i, \delta)\right\| & \leqq g(\delta), \\
\left|e_{0}(\delta)-1\right| & \leqq g(\delta),
\end{aligned}
$$

for $\delta$ sufficiently small.

Recall that $K(i)$ is assumed to have range $r$ based at $i$. Equations (2.11a) and $(2.11 \mathrm{~b})$ then imply by an inductive argument that $\phi_{m, 0}(i)$ has range $\leqq m r$. Consequently for some $c(\delta)$,

$$
\left\|\phi_{0, \Lambda}(i, \sigma, \delta)-\phi_{0, \Lambda}\left(i, \sigma_{j}, \delta\right)\right\| \leqq c(\delta) \delta^{|i-j| / r} .
$$

The same reasoning leads to an estimate on the difference of $\phi_{0, \Lambda}$ 's for different $\Lambda$ 's. We summarize.

Lemma 2.2. Under the assumptions of Theorem 2.1, $\phi_{0, \Lambda}$ satisfies, for $\delta$ sufficiently small,

$$
\begin{gathered}
\left\|\phi_{0, \Lambda}(i, \sigma, \delta)\right\| \leqq c_{0} \delta \\
\text { ii) } \quad\left\|\phi_{0, \Lambda}(i, \sigma, \delta)-\phi_{0, \Lambda}\left(i, \sigma_{j}, \delta\right)\right\| \leqq c(\delta) \delta^{|i-j| / r},
\end{gathered}
$$

and $\phi_{m, 0, \Lambda}(i, \sigma)$ has range $\leqq m r$.

iii) For $\Lambda \subset \Lambda^{\prime}$ and some $c^{\prime}(\delta)$

$$
\left\|\phi_{0, \Lambda}(i, \sigma, \delta)-\phi_{0, \Lambda^{\prime}}(i, \sigma, \delta)\right\| \leqq c^{\prime}(\delta) \delta^{d / r},
$$

where $d$ is the distance between $i$ and the boundary of $\Lambda$.

It remains to define and estimate the $\phi_{n A}$ 's, and $e_{n}$ 's for $n \geqq 1$. It is convenient to define $\tilde{K}(i, \sigma, \delta)$ by

$$
(1+\delta K(i, \sigma)) \exp \phi_{0}(i, \sigma, \delta)=1+\tilde{K}(i, \sigma, \delta) .
$$

Note that $\|K(i, \delta)\|=O(\delta)$. In terms of $\tilde{K}(i, \sigma, \delta)$ the $\phi_{n, \Lambda}$ 's and $e_{n}$ 's are from Eqs. $(2.8 \mathrm{~b})$ and $(2.8 \mathrm{c})$ given by

$$
\begin{gathered}
\sum_{i \in \Lambda}(1+\tilde{K}(i, \sigma, \delta)) \phi_{1}(i, \sigma, \delta)+\sum_{A \subset A} \hat{V}(A) \sigma(A)=|\Lambda| e_{1}(\delta), \\
\sum_{i \in \Lambda}(1+\tilde{K}(i, \sigma, \delta))\left(\phi_{n}(i, \sigma, \delta)+P_{n}\left(\phi_{1}(i, \sigma, \delta), \ldots, \phi_{n-1}(i, \sigma, \delta)\right)\right)=|\Lambda| e_{n}(\delta), \\
n \geqq 2.21
\end{gathered}
$$

Here, for example, $\phi_{1}(i, \sigma, \delta)$ can be obtained as a Neumann series (not in powers of $\delta)$

$$
\begin{aligned}
\phi_{1}(i, \sigma, \delta) & =\sum_{m=0}^{\infty} \tilde{\phi}_{m, 1}(i, \sigma), \\
e_{1}(\delta) & =\sum_{m=0}^{\infty} \tilde{e}_{m, 1}(\delta),
\end{aligned}
$$

with

$$
\sum_{i}\left(\tilde{\phi}_{0.1}(i, \sigma, \delta)-\tilde{e}_{0.1}(\delta)\right)+\sum_{A \subset A} \hat{V}(A) \sigma(A)=0
$$


and otherwise

$$
\sum_{i}\left(\tilde{\phi}_{m, 1}(i, \sigma, \delta)-\tilde{e}_{m, 1}(\delta)\right)+\sum_{i} \tilde{K}(i, \sigma) \tilde{\phi}_{m-1,1}(i, \sigma, \delta)=0, \quad m \geqq 1 .
$$

As before, the solution to these equations are uniquely determined by the requirements that $\phi_{1}(i, \sigma, \delta)$ is homogeneous in $\sigma(i)$ and the $\phi_{1}(i, \sigma, \delta)$ satisfy the closure property. The $\phi_{n}$ 's $n>1$ can be determined similarly.

From Eqs. (2.21a) and (2.21b) and the closure property, we obtain the estimates

$$
\left\|\chi_{1}(i)\right\| \leqq \frac{1}{1-\|\tilde{K}(i)\|} \frac{1}{|\Lambda|} \sum_{A \subset A}|\tilde{V}(A)|
$$

and

$$
\left\|\chi_{n}(i)\right\| \leqq \frac{1+\|\tilde{K}(i)\|}{1-\|\tilde{K}(i)\|} P_{n}\left(\left\|\chi_{1}(i)\right\|, \ldots,\left\|\chi_{n-1}(i)\right\|\right), \quad n \geqq 2
$$

with

$$
\chi_{n}(i, \sigma, \delta)=\phi_{n}(i, \sigma, \delta)-e_{n}(\delta), \quad n \geqq 1 .
$$

Set $a(\delta)=\left\|\chi_{1}(i)\right\|, b(\delta)=(1+\|\tilde{K}(i)\|) /(1-\|\tilde{K}(i)\|)$. Let $f(\varepsilon)$ be the analytic function defined in a neighborhood of $\varepsilon=0[f(0)=0]$ by the implicit relation,

$$
F(f, \varepsilon)=b\left(e^{f(\varepsilon)}-1\right)-(b+1) f(\varepsilon)+a \varepsilon=0 .
$$

As before, substituting

$$
f(\varepsilon, \delta)=f(\varepsilon)=\sum_{n=1}^{\infty} \varepsilon^{n} f_{n}(\delta)
$$

into the rearranged equation

$$
f(\varepsilon, \delta)=f(\varepsilon)=b\left(e^{f(\varepsilon)}-1-f(\varepsilon)\right)+a \varepsilon
$$

gives the non-linear recursion relations,

Again by induction,

$$
\begin{aligned}
& f_{1}(\delta)=a(\delta), \\
& f_{n}(\delta)=b P_{n}\left(f_{1}, \ldots, f_{n-1}\right), \quad n \geqq 2 .
\end{aligned}
$$

$$
\left\|\chi_{n}\right\| \leqq f_{n}(\delta)
$$

so that the series $\sum \varepsilon^{n} \chi_{n}$ and hence the series for $\phi_{\Lambda}(i, \sigma, \varepsilon, \delta), e(\varepsilon, \delta)$, Eqs. (2.6) and (2.7) are absolutely convergent with bounds

$$
\begin{gathered}
\left\|\phi_{A}(i, \sigma, \varepsilon, \delta)\right\| \leqq g(\delta)+f(\varepsilon, \delta), \\
|e(\varepsilon, \delta)| \leqq 1+g(\delta)+f(\varepsilon, \delta),
\end{gathered}
$$

for $\delta, \varepsilon$ sufficiently small, by Ineqs. (2.19a) and (2.19b).

The decay estimates for $\phi_{\Lambda}(i, \sigma, \varepsilon, \delta)$, (ii) and (iii) of the theorem are straightforward but tedious; here we summarize the arguments. Statement (ii) of the lemma, 
and the definition of $\tilde{K}$, Eq. (2.20), imply that if $\tilde{K}(i)$ is expanded,

$$
\tilde{K}(i)=\sum_{m=1}^{\infty} \delta^{m} \tilde{K}_{m}(i),
$$

then $\tilde{K}_{m}(i)$ has range $\leqq m r$. Using this fact in Eqs. (2.21a) and (2.21 b), we obtain inductively the result that $\phi_{m n}(i, \sigma)$, the coefficient of $\delta^{m} \varepsilon^{n}$ in the expansion for $\phi(i, \sigma, \varepsilon, \delta)$, has range $\leqq(n+m) r$. This result along with the fact that $\phi_{0}(i, \sigma, \delta)=O(\delta)$ implies all the estimates of the theorem.

\section{A Large $\varepsilon$-Expansion}

In this section we consider, for illustrative purposes, the particular ferromagnetic Hamiltonian (transverse-Ising)

$$
-H_{\Lambda}(\varepsilon)=\sum_{i \in \Lambda} \sigma^{x}(i)+\varepsilon \sum_{\langle i, j\rangle} \sigma^{z}(i) \sigma^{z}(j)
$$

with $\varepsilon$ large. Here, the second sum is over nearest neighbor bonds. The Hamiltonian is clearly unitarily equivalent to

$$
-H_{\Lambda}^{\prime}(\varepsilon)=\varepsilon \sum_{\langle i, j\rangle} \sigma^{x}(i) \sigma^{x}(j)+\sum_{i} \sigma^{z}(i),
$$

and we will work instead with this Hamiltonian. [It is the unitary transformation taking $H_{\Lambda}(\varepsilon)$ to $H_{A}^{\prime}(\varepsilon)$ which plays the role of a duality transformation.] We again employ the vectors $\{(\sigma)\}$ as an orthonormal basis.

Note first that with respect to the basis $H_{\Lambda}^{\prime}(\varepsilon)$ is reduced; states with an even number or odd number of up spins are mapped invariantly by $H_{A}^{\prime}(\varepsilon)$. For this reason our ansatz for the ground state,

$$
\psi_{\Lambda}^{ \pm}(\varepsilon)=\sum_{\sigma}^{\prime} \exp \left(-\frac{1}{2} \sum_{A} J_{\Lambda}^{ \pm}(A, \varepsilon) \sigma(A)\right)|\sigma\rangle=\sum_{\sigma}^{\prime} \psi_{\Lambda}^{ \pm}(\sigma, \varepsilon)|\sigma\rangle
$$

is a restricted sum over even-up or odd-up states only. [The $J$ 's are not unique since e.g. $\sigma(A)= \pm \sigma(\Lambda-A)$ on even or odd states, respectively.]

As before, we substitute $\psi_{A}^{ \pm}(\varepsilon)$ into the eigenvalue equation,

$$
-H_{\Lambda}(\varepsilon) \psi_{\Lambda}^{ \pm}(\varepsilon)=|\Lambda| e^{ \pm}(\varepsilon) \psi_{\Lambda}^{ \pm}(\varepsilon),
$$

to obtain (again we suppress the subscript $\Lambda$ )

$$
\sum_{\langle i, j\rangle} \exp \left(\phi^{ \pm}(i, \hat{j}, \sigma, \varepsilon)+\phi^{ \pm}(j, \hat{i}, \sigma, \varepsilon)\right)+\frac{1}{\varepsilon} \sum_{i} \sigma(i)=|\Lambda| e^{ \pm}(\varepsilon),
$$

where $\varepsilon|\Lambda| e^{ \pm}(\varepsilon)$ is the eigenvalue, and (for all $i, j \in \Lambda$ )

$$
\phi^{ \pm}(i, j, \sigma, \varepsilon)=\sum_{\substack{A: \vdots \\ i \in A, j \notin A}} J^{ \pm}(A, \varepsilon) \sigma(A) .
$$

In calculations below we observe the conventions that $\sigma(A) \sigma(B)=\sigma(A \Delta B)$, but that $\sigma(\Lambda)= \pm 1$ according to whether $|\Lambda|$ is even or odd. 
Theorem 3.1. For the transverse Ising model defined above, with $i, j$ neighboring, for $\varepsilon$ sufficiently large, there are constants $c_{1}, c_{2}(\varepsilon), c_{3}(\varepsilon), c_{4}(\varepsilon)$ such that

ii)

$$
\begin{gathered}
\left\|\phi_{\Lambda}^{ \pm}(i, \hat{j}, \sigma, \varepsilon)\right\| \leqq c_{1} / \varepsilon, \\
\left\|\phi_{\Lambda}^{ \pm}(i, \hat{j}, \sigma, \varepsilon)-\phi_{\Lambda}^{ \pm}\left(i, \hat{j}, \sigma_{k}, \varepsilon\right)\right\| \leqq c_{2} \varepsilon^{-|i-k|},
\end{gathered}
$$

iii) for $\Lambda \subset \Lambda^{\prime}$ and $i, j \in \Lambda$,

$$
\left\|\phi_{\Lambda}^{ \pm}(i, \hat{j}, \varepsilon)-\phi_{\Lambda^{\prime}}^{ \pm}(i, \hat{j}, \varepsilon)\right\| \leqq c_{3} \varepsilon^{-d},
$$

where $d$ is the distance between $\langle i, j\rangle$ and the boundary of $\Lambda$.

iv) The states $\psi_{A}^{+}, \psi_{A}^{-}$are asymptotically degenerate in the sense,

$$
\left|e^{+}(\varepsilon)-e^{-}(\varepsilon)\right| \leqq c_{4} \varepsilon^{-L},
$$

for $\Lambda$ a cube of sides $L$.

Before proceeding to the proof of this theorem, we show that it implies long range order.

Proposition 3.2. For $\varepsilon$ sufficiently large the ground state of $H_{\Lambda}(\varepsilon)$ exhibits long range order, i.e. there is a constant $k(\varepsilon)>0$ such that

$$
\left\langle\sigma^{z}(k) \sigma^{z}(l)\right\rangle_{\Lambda} \geqq k(\varepsilon)
$$

independent of $\Lambda$ for all $k, l$.

Proof. The expectation in the transformed representation is equal to

$$
\begin{aligned}
\frac{\left\langle\psi_{\Lambda}^{ \pm}(\varepsilon), \sigma^{x}(k) \sigma^{x}(l) \psi_{\Lambda}^{ \pm}\right\rangle}{\left\langle\psi_{\Lambda}^{ \pm}(\varepsilon), \psi_{\Lambda}^{ \pm}(\varepsilon)\right\rangle} & =\frac{\sum_{\sigma}\left(\psi_{\Lambda}^{ \pm}(\sigma, \varepsilon)\right)^{2}\left(\psi_{\Lambda}^{ \pm}\left(\sigma_{k l}, \varepsilon\right) / \psi_{\Lambda}^{ \pm}(\sigma, \varepsilon)\right)}{\sum_{\sigma}\left(\psi_{\Lambda}^{ \pm}(\sigma, \varepsilon)\right)^{2}} \\
& \geqq \inf _{\sigma} \exp \left[\phi_{\Lambda}^{ \pm}(k, \hat{l}, \sigma, \varepsilon)+\phi_{\Lambda}^{ \pm}(l, \hat{k}, \sigma, \varepsilon)\right] \\
& \geqq \exp -\left(\left\|\phi_{\Lambda}^{ \pm}(k, \hat{l}, \sigma, \varepsilon)\right\|+\left\|\phi_{\Lambda}^{ \pm}(l, \hat{k}, \sigma, \varepsilon)\right\|\right) .
\end{aligned}
$$

But the right hand side of this inequality is strictly positive; here, for example,

$$
\begin{aligned}
\left\|\phi^{ \pm}(k, \hat{l}, \sigma, \varepsilon)\right\| & \leqq \frac{1}{2} \sum_{\langle i, j\rangle}\left(\left\|\phi_{\Lambda}^{ \pm}(i, \hat{j}, \sigma, \varepsilon)-\phi_{\Lambda}^{ \pm}\left(i, \hat{j}, \sigma_{k}, \varepsilon\right)\right\|+j, \hat{i} \text {-terms }\right) \\
& \leqq c_{2} \sum_{\langle i, j\rangle}\left(\varepsilon^{-|i-k|}+\varepsilon^{-|j-k|}\right)<\infty
\end{aligned}
$$

by (ii) of the theorem. This concludes the proof of long range order.

Proof of the Theorem. The proof is similar to that of Theorem 2.1, where however, we expand in powers of $1 / \varepsilon$. Writing

$$
\begin{aligned}
\phi^{ \pm}(i, \hat{j}, \sigma, \varepsilon) & =\sum_{n=1}^{\infty} \varepsilon^{-n} \phi_{n}^{ \pm}(i, \hat{j}, \sigma), \\
e^{ \pm}(\varepsilon) & =v+\sum_{n=1}^{\infty} \varepsilon^{-n} e_{n}^{ \pm}
\end{aligned}
$$


(recall $v$ is the dimension of the lattice), we obtain

$$
\sum_{\langle i, j\rangle} \phi_{1}^{ \pm}(i, \hat{j}, \sigma)+\phi_{1}^{ \pm}(j, \hat{i}, \sigma)+\sum_{i} \sigma(i)=|\Lambda| e_{1}^{ \pm}
$$

and otherwise

$$
\begin{aligned}
& \sum_{\langle i, j\rangle \in \Lambda}\left(\phi_{n}^{ \pm}(i, \hat{j}, \sigma)+\phi_{n}^{ \pm}(j, \hat{i}, \sigma)\right. \\
& \left.\quad+P_{n}\left(\phi_{1}^{ \pm}(i, \hat{j}, \sigma)+\phi_{1}^{ \pm}(j, \hat{i}, \sigma), \ldots, \phi_{n-1}^{ \pm}(i, \hat{j}, \sigma)+\phi_{n-1}^{ \pm}(j, \hat{i}, \sigma)\right)\right)=|\Lambda| e_{n}^{ \pm}, \quad n \geqq 2 .
\end{aligned}
$$

These equations are solved just as in Sect. II, but where we observe the convention $\sigma(\Lambda)= \pm 1$, appropriately.

By induction, $\phi_{n}(i, \hat{j}, \sigma)$ has range $\leqq n-1$. Assuming convergence of the series for $\phi^{ \pm}$and $e^{ \pm}$, this fact implies statements (ii) and (iii) of the theorem. It also implies that the system of Eqs. (3.9a) and (3.9b) is the same for the $(+)$ and $(-)$ cases for $n$ sufficiently small so that no $\sigma(\Lambda)$-term occurs, for $n<L$. Hence the expansions for $e^{+}(\varepsilon)$ and $e^{-}(\varepsilon)$ are identical to order $\varepsilon^{-L}$, statement (iv) of the theorem.

Regarding convergence, we set

$$
\chi_{n}^{ \pm}(i, j, \sigma)=\phi_{n}^{ \pm}(i, \hat{j}, \sigma)+\phi_{n}^{ \pm}(j, \hat{i}, \sigma)-e_{n}^{ \pm} / v
$$

Note that

$$
\left\|\chi_{n}^{ \pm}(i, \hat{j})\right\|=\left\|\phi_{n}^{ \pm}(i, \hat{j})\right\|+\left\|\phi_{n}^{ \pm}(j, \hat{i})\right\|+\left|e_{n}^{ \pm} / v\right| .
$$

From Eqs. (3.9a) and (3.9b), we have that

$$
\left\|\chi_{1}^{ \pm}(i, j)\right\| \leqq 1 / v
$$

and

$$
\left\|\chi_{n}^{ \pm}(i, j)\right\| \leqq P_{n}\left(\left\|\chi_{1}^{ \pm}(i, j)\right\|, \ldots,\left\|\chi_{n-1}^{ \pm}(i, j)\right\|\right), \quad n \geqq 2 .
$$

By the same argument used in Sect. II,

$$
\left\|\chi_{n}^{ \pm}(i, j)\right\| \leqq h_{n}
$$

with

$$
h(z)=\sum_{n=1}^{\infty} z^{n} h_{n},
$$

defined in a neighborhood of 0 by the implicit relation $(h(0)=0)$

$$
2 h(z)-e^{h(z)}+1-z / v=0 .
$$

In particular, for $\varepsilon$ sufficiently large

$$
\begin{aligned}
&\left\|\phi^{ \pm}(i, \hat{j}, \sigma, \varepsilon)\right\| \leqq h(1 / \varepsilon), \\
&\left|e^{ \pm}(\varepsilon)-v\right| \leqq v h(1 / \varepsilon) .
\end{aligned}
$$

This concludes the proof of the theorem. 


\section{References}

1. Klein, A., Landau, L. J.: Stochastic processes associated with KMS states. J. Funct. Anal. 42, 368-428 (1981)

2. Hagedorn, R., Rafelski, J.: Analytic structure and explicit solution of an important implicit equation. Commun. Math. Phys. 83, 563-578 (1982)

3. Pfeuty, P.: The one-dimensional Ising model with a transverse field. Ann. Phys. N.Y. 57, 79-90 (1970)

4. Pfeuty, P., Elliot, R. J.: The Ising model with a transverse field. II. Ground state properties. J. Phys. C 4, 2370-2385 (1971)

5. Kadanoff, L. P., Kohmoto, M.: Quantum mechanical ground states, nonlinear Schrödinger equations and linked-cluster expansions. J. Phys. A 14, 1291-1305 (1981)

6. Ginibre, J.: Existence of phase transitions for quantum Lattice systems. Commun. Math. Phys. 14, 205-234 (1969)

7. Fröhlich, J., Lieb, E.: Phase transitions in anisotropic spin systems. Commun. Math. Phys. 60, 233-267 (1978)

8. Kirkwood, J. R.: Ph.D. dissertation, University of Virginia (1982)

9. Ruelle, D.: Statistical mechanics. New York: Benjamin 1969

Communicated by J. Fröhlich

Received November 3, 1982 